\title{
Time Slot Switching for Integrated Services in Fiber Optic PBX/LAN
}

\author{
MING-KANG LIU, MEMBER, IEEE, DAVID G. MESSERSCHMITT, FELLOW, IEEE, AND DAVID A. HODGES, FELLOW, IEEE
}

\begin{abstract}
A new medium access protocol called time slot switching (TSS) is proposed for use in optical fiber local area networks. This protocol incorporates features of time division, space division, and time compression for users to share a common medium. VLSI CMOS electrical crosspoints are used to switch traffic within individual time slots. With these features, data, voice, and video services can all be combined in a single network, In addition, the speed of the electronics can be maximized to match the available optical bandwidth.

Operational principles of the TSS protocol are explained. A performance analysis is presented to show the tradeoffs among traffic capacity, frame guard time, blocking probability, and transmission delay. The analysis is done for $64 \mathrm{Kbit} / \mathrm{s}$ channels, and the results show that TSS is more attractive than broadcast protocols for voice traffic or constant-rate data traffic. An approach to integrating voice, data, and video traffic within TSS is also described.
\end{abstract}

\section{INTRODUCTION}

OCAL area networks (LAN's) were first used to provide Ldata communication among computers. A review of LAN architectures can be found in [1]. A major goal now is to integrate all data, voice, and video traffic in a single LAN optimized for economics and performance. There have been many proposals to integrate voice and data services in LAN's [2]-[6], and they primarily incorporate packet switching. This paper presents a circuit switching access protocol that uses wide-band circuit switches compatible with optical fiber technology and provides the desired traffic integration.

Fiber optics is an emerging technology that provides abundant bandwidth (a few Gbits/s) at low cost. To provide economical optical fiber LAN systems, the electronic functions requircd in transmittcrs, reccivers, multiplexers, message switches, etc., should all be simplified to match the speed of optical fiber transmission. Consequently, we have chosen circuit switching primarily because it allows a switching fabric that does not actually examine or process the bit streams passing through (as we show later), with the result that the bit rate can be considerably greater for a given low-cost electronic technology. This circuit switching approach is supported by the recent work in [9]-[10], which has demonstrated the feasibility of economical electronic circuit switches in CMOS VLSI, operating at up to $200 \mathrm{Mbit} / \mathrm{s}$ data rates per channel.

Circuit switching has been used in LAN's at $1 \mathrm{Mbit} / \mathrm{s}[7]$ and $380 \mathrm{Mbits} / \mathrm{s}$ [8]. Also, many extensions of circuitswitched PBX's to provide both data and voice services have also been made, e.g., NEC NEAX 2400, AT\&T-IS System

Paper approved by the Editor for Communication Networks of the IEEE Communications Society. Manuscript received July 31, 1986; revised June 15,1987 . This work was supported by grants from Advanced Micro Devices, Fairchild Semiconductor, Harris Semiconductor, National Semiconductor, Intel Corporation, and Bell Communications Research, with a matching grant from the University of California MICRO program.

M. -K. Liu is with Bell Communications Research, Red Bank, NJ 07701.

D. G. Messerschmitt and D. A. Hodges are with the Department of Electrical Engineering and Computer Sciences and the Electronics Research Laboratory, University of California, Berkeley, CA 94720

IEEE Log Number 8928210.
75, Honeywell ASBU 501, and ROLM CBX II [11]-[14]. In their approaches, data and voice are time division multiplexed (TDM) to share circuit switches. In this paper, a variant of circuit switching called time slot switching (TSS) is proposed. Distinctive features of TSS are that 1) circuit switches are wide-band (a few hundred Mbits/s) and 2) different channels share common switching by time division multiple access (TDMA) [15], a media access protocol for which no synchronization between users is needed.

TSS operational principles will be explained in Section II, limited to the case of one type of constant-rate traffic. The switching management for TSS is presented in Section III. Network performance is analyzed in Section IV. Finally, Section $V$ describes an approach to integrating voice, data, and video using TSS.

\section{HOMOGFNFOUS TSS NETWORKS}

By a "homogeneous" TSS network, we mean that all supported circuits have the same bit rate (e.g., $64 \mathrm{Kbit} / \mathrm{s}$ PCM voice); this homogeneous assumption will be relaxed in Section $\mathrm{V}$.

\section{A. Network Topology and Architecture}

A TSS network generally consists of switching nodes connected by optical fiber links. The switching nodes support simultaneous circuits by space-division switches. Users access the switching nodes through concentrators called time compression multiplexers (TCM), ' which multiplex user traffic in a time-division fashion, as shown in Figs. 1, 2.

Fig. I illustrates a network structure with two types of switching nodes. Type $A$ switches are only connected with other switching nodes, and type $B$ switches connect TCM's with type $A$ and/or other type $B$ nodes. Within each switching node, there is a spatial circuit switch which cross-connects the traffic in the network.

\section{B. Basic Operations}

Time slot switching (TSS) can be described as a switched time-division multiple-access (TDMA) network [15]. TDMA is often used in satellite networks for the same reason it is used here; namely, it allows very simplc hardware, and hence the maximum speed for a given implementation technology. TCM's are used in TSS to give users TDMA access. In addition to the TCM's, TSS incorporates circuit switches, which allow multiple transmissions simultaneously; therefore, a higher traffic throughput can be achieved as compared to shared-medium broadcasting protocols such as carrier sense multiple access (CSMA).

To set up a connection path, both the TCM's and the switches should agree to a common time-slot definition, as shown in Fig. 2. A transmitted user bit stream is first multiplexed by a TCM, followed by a series of circuit switch cross-points correctly arranged in a certain time slot, and is finally demultiplexed at the destination TCM during the same

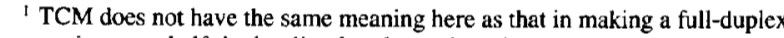
connection on a half-duplex line by alternating directions of transmission. 


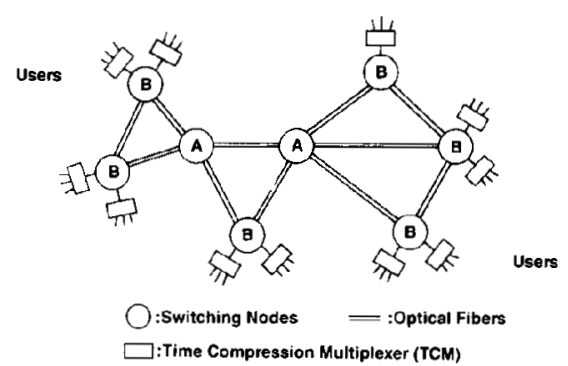

Fig. 1. An illustration of the time slot switching network topology where there are two types of switching nodes. Type $B$ is connected to TCM's, while Type $A$ is not.

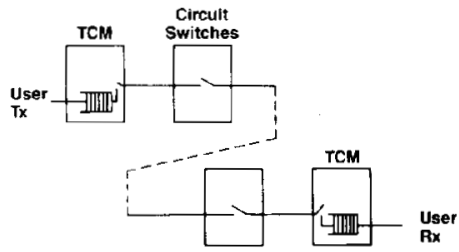

A Complete Circuit Link At One Time Sio

(a)

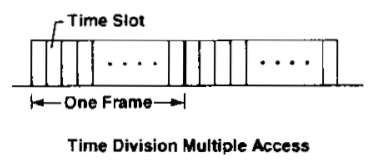

(b)

Fig. 2. Illustration of circuit connections in a time slot switching network. (a) Spatial connections at a given time slot. (b) Time domain access: TDMA.

time slot. The required slot timing synchronization of TCM's and switches is established by prearrangement and will be discussed in Section III.

The TCM is an interface between low-speed users and highspeed circuit switches. The TCM performs two functions. First, the TCM buffers input/output for one frame duration for low-speed users. Second, the TCM transmits or receives these bits within the appropriate time slot for which the desired connectivity has been prearranged through the space-division network. The same time slot in each frame is dedicated to the connected circuit until the call finishes. Typically, a TCM will handle several circuits simultaneously, so each circuit has its own buffer. During each time slot, the data in the appropriate buffer is transmitted over the high-speed link. Similarly, on the receiving side, data arriving on a high-speed link within each time slot is stored in the appropriate receiving buffers, and then transmitted at a lower speed to the user terminals. To summarize, a lower speed circuit is compressed and transmitted over the higher speed channel in a specific time slot in the frame, and at the receiver, the circuit is decompressed for that same time slot to the lower speed user terminal.

The Circuit Switches provide the physical paths for different connections. The circuit switch has an associated slot memory which contains the connection information used for changing the configuration of the cross-point matrix after each time slot. In each time slot, there are simultaneous circuits being transmitted by the switches (in different spatial paths), each at a very high speed (e.g., $200 \mathrm{Mbits} / \mathrm{s}$ ) compared to that of users. This multiple circuit configuration achieves a total network traffic capacity much greater than that achieved by broadcasting protocols such as token passing or CSMA operating at the same speed on each link. The circuit switch in TSS does not examine the bit streams and consequently does not need timing recovery circuits. This not only avoids costly
TABLE I

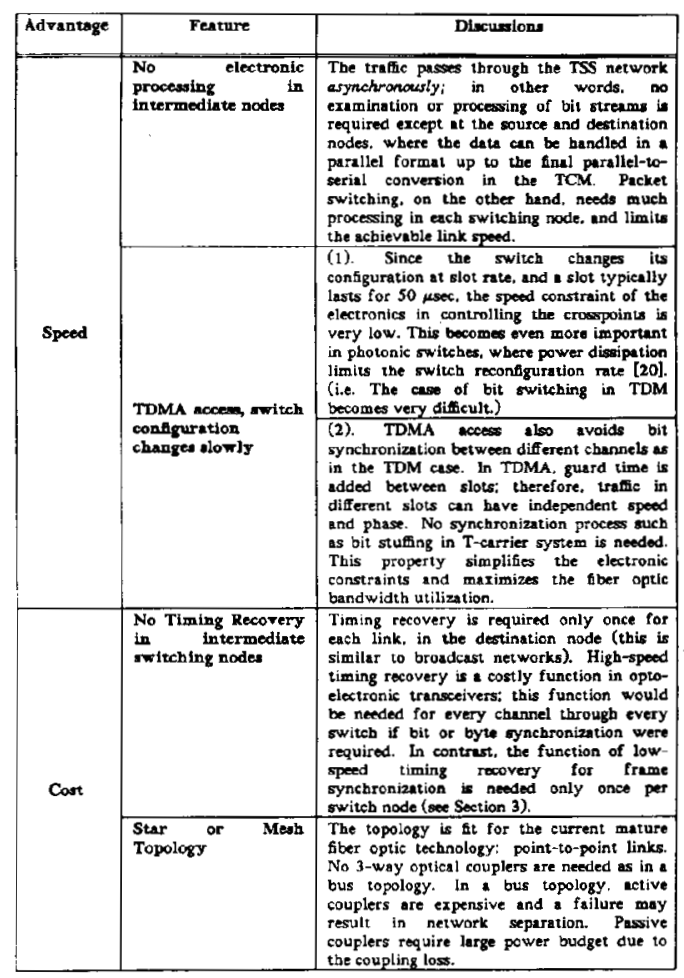

implementation of high-speed timing recovery, but also allows different speeds of traffic to coexist in the same network.

\section{Some Remarks on TSS}

For the foreseeable future, electronics will limit the practical bandwidth of optical fiber networks. TSS attempts to minimize the electronic constraints to fully take advantage of optical fiber technology, as summarized in Table I.

TSS also has other advantages, for example, collisions in CSMA are eliminated by the arbitration provided by a central controller, and circuit switching results in a deterministic delay, which is desirable in voice traffic and some types of data communications. In addition, because the asynchronous traffic flow within each time slot, different instantaneous bit rates can coexist within the same network. For example, the logically separate control network can operate at a lower speed within a dedicated time slot, eliminating the necessity for using the same high-speed electronics technology in the implementation of the control as compared to the switching function itself.

Some disadvantages of TSS include the following.

1) Guard Time: Though the switching nodes receive the same global timing, they are spatially distributed in the network. Timing skew in the synchronization of time slots among these switching nodes is inevitable. This, coupled with the propagation delay of signals through the network results in the need for guard times in each time slot, and a resultant reduction in traffic capacity. This is similar to the requirement for guard times in TDMA satellite transmission with distributed earth stations. This guard time is analyzed in detail in Section IV-A, which shows the time is proportional to the network size. Therefore, the need for reasonable throughput efficiency limits the geographical size of the network, although this limitation can be circumvented by using gateways with internal buffering.

2) Circuit Blocking: Circuit switching results in blocking of connections during high traffic loads [16]. At a given traffic 
intensity and time slot length, the blocking probability can be reduced by increasing the total number of time slots in a frame, but at the expense of a larger frame size which results in a larger transmission delay. The frame size cannot be arbitrarily large [see eq. (9)]; however, within its possible range, an achievable traffic intensity level can be estimated by specifying the blocking probability (e.g., $10^{-3}$ ), (7)

3) Buffering Delay: TSS results in a buffering delay. This is due to the need to 1) store one time slot of voice samples (e.g., 64 Kbits/s PCM) in the transmitting TCM buffer before the transmission to the higher speed network, and 2) store the voice samples in one time slot in the destination TCM buffer at link speed (e.g., $200 \mathrm{Mbits} / \mathrm{s}$ ). Since the buffering delay in the transmitting TCM dominates (one frame), only this delay (called compression delay) is considered in the analysis of Section IV-C. This presents a problem in voice and interactive applications.

4) Timing Jitter: Timing jitter is introduced not only in optical fibers and transceivers, but also in each space-division switch because of crosstalk and dc offset. Without retiming in intermediate switches, timing jitter will accumulate and eventually cause unacceptable bit error rates. Consequently, the achievable number of intermediate switching nodes without retiming is limited by this jitter accumulation. Preliminary experiments to quantify this limit [21], [22] indicate that for the local area network application the jitter in each switch is small, and the jitter accumulation through tens of switching nodes is still insignificant.

5) Central Controller: The circuit switching architecture in TSS needs a central controller to set up circuit links to provide a global slot timing. This suggests that the network is vulnerable to single-point failure. This problem is encountered in standard PBX and telephone switches, and is typically controlled through redundant controllers.

\section{SWitChing MANAGEMENT}

Because TSS has both time and space division switching, the TCM's and switches need 1) frame timing, 2) slot timing, 3) cross-point connection information, and 4) TCM multiplexing information to properly set up and tear down connections.

\section{A. Frame and Slot Timing}

The frame and slot timing are needed to change the TCM and switch configurations. These configurations are different in each time slot, and repeat each frame.

The frame timing can be obtained simply by broadcasting a frame header of a special pattern and frequency from the central controller to every switching node, similar to TDMA as used in satellite communications [15]. In TSS, this frame header can be put at the beginning of each frame, and by sensing this particular pattern, the frame timing can be extracted. Since both the frame and slot timing are at relatively low frequency (e.g., $20 \mathrm{~Hz}$ if each frame is of $50 \mathrm{~ms}$, and 20 $\mathrm{KHz}$ if each slot is of $50 \mu \mathrm{s}$ ), a simple PLL and a frequency divider can generate slot timing synchronized with respect to the frame timing, Fig. 3 .

\section{B. Circuit Connection Setup}

The switches and TCM's need connection information to set up circuits correctly in each time slot. Since the switching nodes are generally distributed in the network, this connection information is determined and distributed by the central controller after the controller receives "call requests" or "call terminations" from users. This section describes an algorithm for the controller to set up circuits, and the next subsection suggests how to pass the connection information between the switches and controller.

First, the central controller has a cross-point allocation table for each circuit switch and each time slot. After receiving a circuit request from one switching node to another node, the

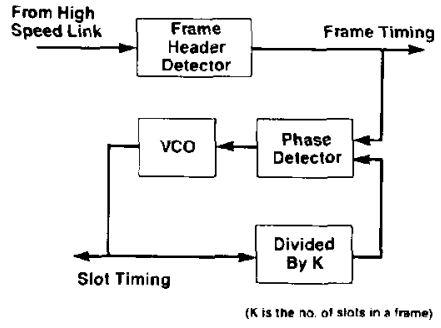

Fig. 3. Illustration of the global timing recovery by a phase lock loop (PLL).

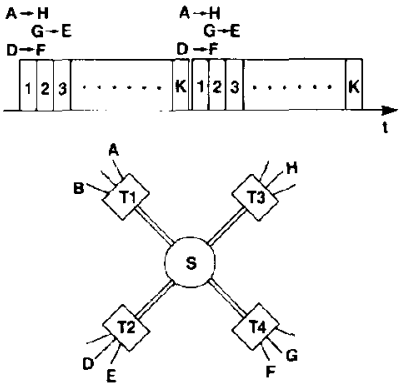

Fig. 4. An example of the time slot switching operation. The network has a single star topology, connected by four TCM's. Initially, user $A$ is talking to $H$ through TCM's $T 1$ and $T 3$.

controller linearly searches for the first available time slot from the beginning of the frame. Generally, there may be morc than one possible circuit path available for a call, in which case switches and cross-points of the minimum distance path will be chosen. There may exist other search algorithms which produce a shorter search time, but a detailed study of these algorithms is beyond the scope of this paper.

In the example illustrated in Fig. 4, user terminal $A$ is currently transmitting to user $H$ during time slot 1 of each frame. TCM's $T 1, T 3$, and the switch $S$ are synchronized to provide this connection. Now, user terminal $D$ wants to transmit to user $F$. There is no other traffic through TCM $T 2$ and $T 4$, and the current link between $A$ and $H$ causes no conflict in using circuit switch $S$ for this new request. Thus, the central controller can assign any slot to this new request, and it chooses slot 1 by this algorithm. There will be two independent circuits in the same time slot. A few moments later, user terminal $G$ wants to communicate with user $E$ and sends the call request to the central controller. There is a conflict with the existing circuits in the central circuit switch $S$, and $T 2$ and $T 4$ are reserved for $D-F$ at slot no. 1 . Consequently, the central controller will assign time slot 2 for this new call.

If there is neither an available time slot nor an available space-division path for a new requested circuit when the destination is free, the call is blocked. The probability of blocking is estimated in Section IV. Any circuit can be terminated by transmitting an appropriate "disconnect" message to the central controller, releasing the associated time slot for future use.

\section{The Control Traffic}

A logically separate signaling network is needed to provide the connection information as mentioned above. The amount of this signaling traffic can be estimated as follows. Suppose therc are 1000 users with active probability 50 percent (i.e., 50 percent of users on the average are using circuits at any time), and the average circuit holding time is three minutes such as in voice conversation, then there will be only 
$1000 \cdot 0.5 / 180=2.8 \mathrm{calls} / \mathrm{s}$. Suppose each circuit establish ment requires $1 \mathrm{Kbit} / \mathrm{s}$ call $^{2}$, then the total control traffic demand is only about $3.0 \mathrm{Kbit} / \mathrm{s}(\approx 2.8 \mathrm{calls} / \mathrm{s} \cdot 1 \mathrm{Kbit} / \mathrm{s}$ call $)$.

As mentioned before, although this control traffic can be supported by a separate lower speed network, it can be supported by the same TSS network by reserving the first few slots of a frame. For example, in the network shown in Fig. 1, if the switching node of type $A$ on the right is the central controller, the connection information for all the switches and TCM's can be broadcast during the second time slot of each frame, and the call request and disconnect information from all the switching nodes of type $B$ can be sent to the controller during the third and fourth time slots (at least two slots are needed since there is a circuit conflict of two type $B$ switching nodes on the left). The signaling uses slot two because the first slot is already assigned for the extraction of frame timing.

\section{Performance Analysis of Homogeneous tSS}

In this section, we present a quantitative analysis of the behavior of the TSS network. For simplicity, we treat the homogeneous case in which all circuits have the same bit rate.

In the analysis of TSS, we must consider a number of dependent parameters: the number of time slots per frame, the length of a time slot, the blocking probability for new circuit connection requests, traffic utilization, and the compression delay. In the remainder of this section, we determine the following.

1) The guard time, which is required to protect data in adjacent slots. It will be shown to be proportional to the maximum propagation delay in the network.

2) The blocking probability, which is a function of the network topology and available circuits. We approximate this probability for a single or double star network topology.

3) The compression delay, which depends on the total number of time slots per frame, the slot size, and hence the traffic utilization. This will allow us to study the tradeoff between network utilization and the compression delay for a fixed blocking probability and network topology.

Before proceeding with the analysis, some definitions are appropriate.

Network Circuits: The average number of circuits connected at one time. Using telephone terminology, this has the units of Erlangs where one Erlang is equivalent to one circuit continuously connected.

Network Utilization: The ratio of average total network throughput (bits per second) to the capacity of one link. Since multiple links are utilized in TSS due to switching, the network utilization in general can be greater than 100 percent. This definition of utilization enables us to compare the traffic capacity to that of a multiple access protocol using a single shared link with the same capacity.

Blocking Probability: The probability that a new call request will be denied due to the absence of an available circuit path for any time slot between source and destination.

Compression Delay: As explained in Section II, this is the buffer delay to store voice samples in the transmitting TCM buffer for one frame period before its transmission to the network. There are other delays constituting the total transmission delay, for example, the propagation delay and the buffer delay in the destination TCM buffer. They are comparatively smaller and therefore are not considered in the following analysis.

\section{A. Guard Time Analysis}

A time slot can be decomposed in three sequential parts: 1)

\footnotetext{
${ }^{2}$ These 1 Kbits include the information to send source and destination addresses to the controller, and to send the connection information to the switching nodes.
}

the leading guard time $\left.t_{L}, 2\right)$ the maximum available interval for data transmission $t_{D}^{\mathrm{MAX}}$, and 3) the trailing guard time $t_{T}$. The interval of time corresponding to a time slot is

$$
t_{\text {slot }}=t_{L}+t_{D}^{\mathrm{MAX}}+t_{T} \equiv t_{D}^{\mathrm{MAX}}+t_{\mathrm{idle}}
$$

where the idle time is defined $t_{\text {idl }}=t_{L}+t_{T}$. The requirement for nonzero $t_{L}$ and $t_{T}$ derives from finite propagation delay and timing skew (due to different global timing propagation delays between the central clock and switching nodes in the network).

Within the maximum available interval $t_{D}^{\mathrm{MAX}}$, if $t_{D}$ is really used for transmission $\left(t_{D} \leq t_{D}^{\mathrm{MAX}}\right)$, we can define the slot utilization $\eta_{\text {slot }}$ to be

$$
\eta_{\text {slot }} \equiv \frac{t_{D}}{t_{\text {slot }}} \leqslant 1-\frac{t_{\text {idle }}}{t_{\text {slot }}} .
$$

From (2), we want to minimize the guard times $t_{L}$ and $t_{T}$. The minimal condition of $t_{L}+t_{T} \equiv t_{\text {idle }}$ can be obtained as shown in Fig. 5. Starting with $t_{L}$, the purpose of the leading guard time is to ensure that a packet does not arrive at a node prior to the start of a time slot. Suppose node $A$ sends a packet to node $B$ where the global time slot timing of node $A$ leads that of node $B$, as shown in Fig. 5(a). To ensure that the beginning of the packet from node $A$ does not arrive too early, we have

$$
t_{L}+t_{p g, A B} \geqslant t_{\Delta, A B}
$$

where $t_{p g, A B}$ is the propagation delay between nodes $A$ and $B$, and $t_{\triangle, A B}$ is the timing skew for the time slot clock between nodes $A$ and $B$.

Similarly, the purpose of the trailing guard time is to ensure that the end of a packet from node $B$ to node $A$ occurs before the end of the time slot as shown in Fig. 5(b). The necessary condition is

$$
t_{T} \geqslant t_{p g, A B}+t_{\triangle A B}
$$

The above conditions have to hold for any two switching nodes, so we have

$$
t_{L}=\left\{\begin{array}{l}
0, \quad \text { if } t_{\Delta, \max }<t_{p g, \min } ; \\
t_{\Delta, \max }-t_{p g, \min }, \quad \text { otherwise }
\end{array}\right.
$$

where $t_{\Delta, \max }$ is the maximum timing skew, and $t_{p g, \text { min }}$ is the minimum propagation delay between switching nodes in the network. Also,

$$
t_{T} \geqslant t_{\Delta, \max }+t_{p g, \max } .
$$

The worst case occurs when $t_{p g, \min }=0$ and $t_{\Delta, \max } \approx t_{p g, \max }=$ $t_{p g}$, in which case

$$
\begin{gathered}
t_{L}=t_{p g}, \\
t_{T} \approx 2 t_{p g}, \\
t_{\mathrm{idle}} \equiv t_{L}+t_{T} \approx 3 t_{p g} .
\end{gathered}
$$

Because $t_{p g}$, the maximum propagation time through the network, cannot be reduced without compromising the size of the network, the only way to minimize the guard time $t_{\text {idle }}$ is to minimize the timing skew $t_{\Delta, \max }$. We can borrow a technique from TDMA satellite communications, where guard times also exist because of distributed earth stations [15]. In this environment, the guard time can be reduced by estimating the distance between the earth station and the satellite when the switching nodes are initialized. Similarly, the propagation delay between a switching node to the central controller in TSS can be estimated, and the frame timing can be offset by this amount. That is, the time skew effect in the guard time can be 

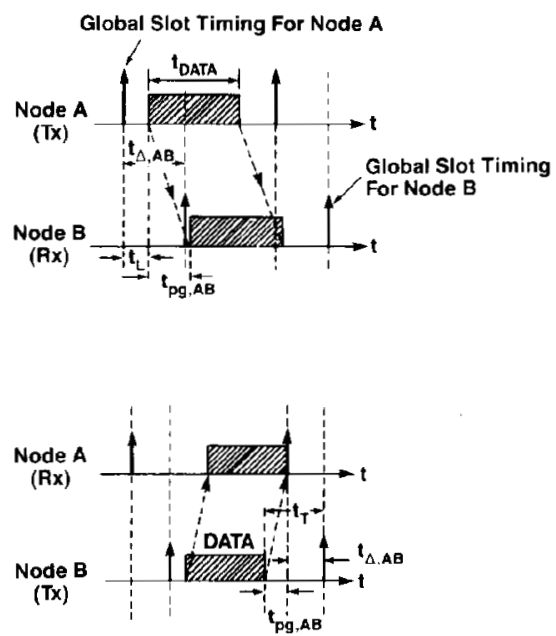

Fig. 5. Guard time analysis in the time slot switching network. (a). The worst case for the leading guard time. (b). The worst case for the trailing guard time.

reduced to a minimum; in that case, by (3)-(4), $t_{L}$ will be approximately 0 and $t_{T}$ will be only $t_{p g}$. The idle time could be reduced to

$$
t_{\text {idle }} \approx t_{p g} \text {. }
$$

In particular, if there is only one switching node in TSS, as in the case where many earth stations communicate to each other by only one satellite, the propagation effect in the trailing guard time [eq. (4)] will also be zero, and the guard time can be made to approach zero.

\section{B. Network Circuits and Blocking Probability}

In this section, we calculate the relationship between the average number of active circuits and the blocking probability in TSS. For a given network topology, any TSS network can be transformed into a topologically equivalent space-division switching network for which the blocking probability can be estimated [16]. To illustrate, a simple single star network in Fig. 4 is considered. In general, there are $\boldsymbol{M}$ TCM's connected to the central $M$ by $M$ switch, $K$ time slots in each fame, and $N$ user terminals connected to each TCM. As shown in Fig. 6, this switching network is equivalent to a space-division network with three stages. There are $K(M$ by $M)$ switches in the middle stage, one for each time slot, corresponding to the single physical time-divided space-division $M$ by $M$ switch. Each TCM allows the $N$ user terminals to access one of $K$ time slots, and hence is topologically equivalent to an $N$ by $K$ space-division switch.

In general, except for special designs [17], switching networks have a nonzero blocking probability. Given a circuit switch structure, Lee [16] developed an approximate method of evaluating the blocking probability. For a single-star network shown in Fig. 4, Lee's method (see Appendix A), yields the following approximation to the blocking probability:

$$
\begin{gathered}
P_{b k}=\left\{1-(1-p)^{2}\right\}^{K}, \\
p=\frac{A}{M K}
\end{gathered}
$$

where $A$ is the total offered traffic in Erlangs.

A similar analysis for a double-star network (see Appendix B) gives

$$
A \approx K N_{2}\left\{1-\left(1-P_{b k}^{1 / K}\right)^{1 / 2}\right\}
$$
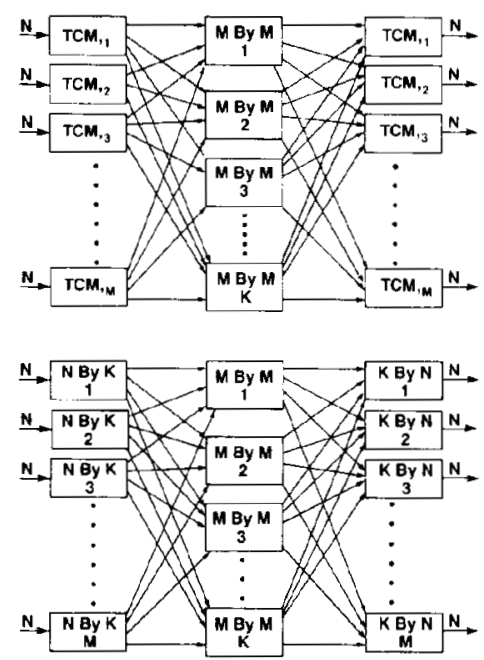

Fig. 6. (a) Logical connections for the single star topology in Fig. 4. Each $M$ by $M$ switch in the middle corresponds to one time slot in the frame. (b) Equivalent three stage space-division switching network.

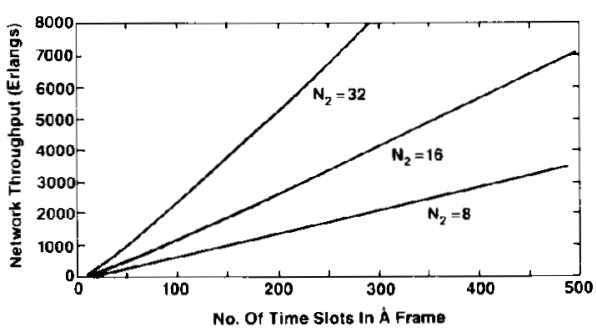

Fig. 7. Network throughput as a function of the total number of time slots in a frame and the size of the circuit switch. The result is based on the method of C. Y. Lee [1] where the blocking probability is fixed at $10^{-3}$.

where $N_{2}$ is the number of substars which are connected to the central star. Fig. 7 illustrates this relation numerically for a blocking probability of $10^{-3}$, similar to the blocking probability requirements in the telephone network.

Our experience is that Lee's method yields an estimate higher than the actual blocking probability. This is substantiated by simulation, the results of which are compared to (7) in Figs. 8 and 9 . We will use (7) in the following performance analysis.

\section{Compression Delay}

The compression delay in TSS is equal to the duration of one frame. Since there are $K$ time slots in each frame, the compression delay is

$$
D=K t_{\text {sior }}
$$

If each circuit corresponds to one time slot,

$$
R t_{D}^{\mathrm{MAX}} \geqslant B D=R t_{D},
$$

ensures that the input bit rate can be accommodated within one time slot excluding the guard time where $R$ is the bandwidth on a link, $B$ is the bit rate for one circuit, $t_{D}$ is the time interval for information transmission, and $D$ is the duration of a frame from (8). The left side of (9) equals the maximum available number of bits for transmission during one time slot, while the right side represents the number of bits accepted for one circuit from the data terminal. Equality occurs when $t_{D}^{\mathrm{MAX}}$ is completely used for transmission. 


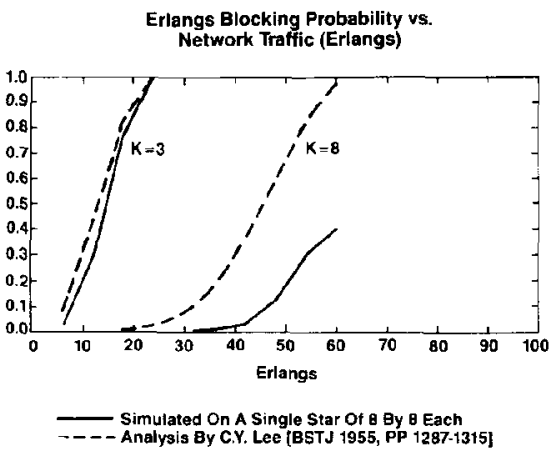

Fig. 8. A comparison of the blocking probability calculation obtained by simulation and Lee's method for the single star topology.

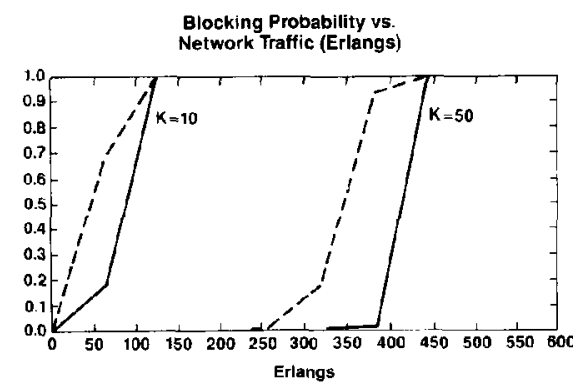

\section{--- Simulated On A Double Star O18 8y 8. Each}

Fig. 9. A comparison of the blocking probability calculation obtained by simulation and Lee's method for the double star topology.

By simple manipulation of (1), (8), (9),

$$
t_{\text {slot }}=\frac{t_{\text {idle }}+\Delta t_{D}}{1-\frac{K B}{R}}
$$

where $\Delta t_{D}=t_{D}^{\mathrm{MAX}}-t_{D}$ and using (8),

$$
D=K \frac{t_{\text {idle }}+\Delta t_{D}}{1-\frac{K B}{R}} .
$$

Since larger $K$ results in a larger number of circuits available at a given blocking probability [eq. (7)], we see the expected tradeoff between throughput and delay where the delay increases with throughput. In addition, $K B$ is the total available throughput passing through any cross-point and is limited by the link bandwidth $R$; as a result, $K$ cannot be arbitrary large and is bounded by $R / B$. Also, $\Delta t_{D}$ cannot be reduced to zero in practice, since some preamble bits are neccssary for the bit timing recovery at the receiver. However, it is much smaller than $t_{\text {idle }}$ and can be neglected.

Fig. 10 gives numerical results describing these relations for $B=64 \mathrm{Kbits} / \mathrm{s}$. We expect that $R=200 \mathrm{Mbits} / \mathrm{s}$ can be achieved using a low-cost CMOS technology for the switches [9], [10], but to be conservative we use $R=100 \mathrm{Mbits} / \mathrm{s}$. In the figure, $\Delta t_{D}$ in (11) is assumed to be 0 . If a propagation velocity of $2 \cdot 10^{8} \mathrm{~m} / \mathrm{s}$ is assumed in the optical fiber and the worst case in (5) is assumed, then the ratio of $t_{\text {idle }}$ to network size is $15 \mu \mathrm{s} / \mathrm{Km}$ in Fig. 10. The three cases shown correspond, therefore, to a maximum network dimension of 1 , 2 , and $3 \mathrm{Km}$.

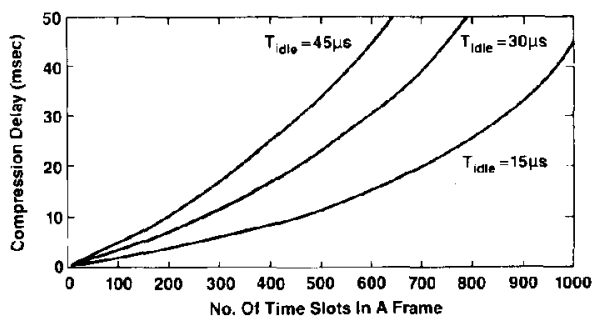

Fig. 10. Compression delay as a function of the total number of time slots in a frame, and the size of the network. For a $2 \cdot 10^{8}$ meters $/ \mathrm{s}$ propagation velocity, and three times the maximum propagation delay for the total guard time in each time slot, each $15 \mu$ s corresponds to a $1 \mathrm{Km}$ network maximum dimension. $64 \mathrm{Kbits} / \mathrm{s}$ per time slot and a link bandwidth of $100 \mathrm{Mbits} / \mathrm{s}$ are assumed.

\section{Network Utilization Versus Compression Delay}

The previous results can be used to obtain the relationship between network utilization and compression delay. By (2) and $(10)$, we have

$$
\eta_{\text {slot }}=\frac{K B}{R}
$$

Furthermore, total network utilization can be easily expressed in terms of the average offered traffic in Erlangs $\boldsymbol{A}$. Because there are $A$ circuits on average, by definition of network utilization, we have

$$
\eta_{\mathrm{network}}=\frac{A B}{R}=\frac{A}{K} \eta_{\text {slot }} .
$$

By (7), we have

$$
\eta_{\text {network }} \approx N_{2}\left\{1-\left(1-P_{b k}^{1 / K}\right)^{1 / 2}\right\} \eta_{\text {slot }} .
$$

Equation (14) gives very important physical insight into TSS networks. Total network utilization is naturally increased by the factor $\mathrm{N}_{2}$ due to the multiple simultaneous connections, while it is subject to two degrading factors. One is the effect of the guard time expressed by $\eta_{\mathrm{slo}}$, and the other is the blocking probability limitation in the brackets.

By combining (11) with (12) and (14), an expression relating $D$ and $\eta_{\text {network }}$ can be obtained. Observing that

$$
\alpha \equiv 1-\left(1-P_{b k}^{1 / K}\right)^{1 / 2}
$$

is a weak function of $K$, simple manipulation of (7), (11), and (13) gives

$$
D=\frac{R\left(t_{\mathrm{idle}}+\Delta t_{D}\right)}{B} \frac{\frac{\eta_{\text {network }}}{N_{2} \alpha}}{1-\frac{\eta_{\text {network }}}{N_{2} \alpha}} .
$$

This expression is very similar to those in multiple access protocols, even though the underlying reasons are quite different. In multiple access protocols, the delay-utilization tradeoff is due to the larger queuing delay at the higher network utilization; in TSS, the tradeoff (15) is due to the larger compression delay needed to maintain a fixed blocking probability at the higher utilization. Fig. 11 , with $\Delta t_{D}$ assumed zero, shows that $\eta_{\text {retwork }}>1$ can be achieved at a reasonable delay; that is, the total utilization can be much larger than the bandwidth of one link that would be characteristic of a multiaccess protocol. For example, at a $50 \mathrm{~ms}$ compression 


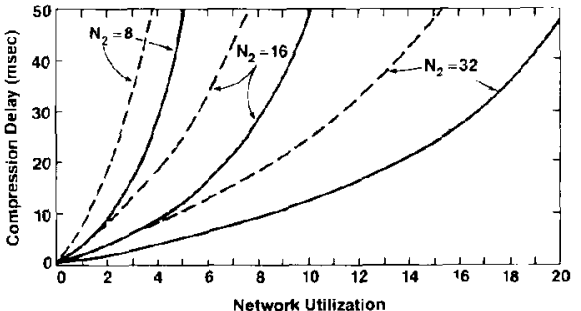

Fig. 11. Tradeoffs between the compression delay and network utilization. This is under the condition of $64 \mathrm{Kbits} / \mathrm{s}$ per time slot, blocking probability of $10^{3}$, and link bandwidth $100 \mathrm{Mbits} / \mathrm{s}$. Solid lines correspond to a network dimension of $1 \mathrm{Km}$, while dash lines correspond to $2 \mathrm{Km}$.

delay, $100 \mathrm{Mbit} / \mathrm{s}$ network bandwidth, $10^{-3}$ blocking probability, and a 16 by 16 switch in the central star of a double star network, $\eta_{\text {network }} \approx 10$ is easily achieved for $64 \mathrm{Kbits} / \mathrm{s} / \mathrm{slot}$. This corresponds to a $1000 \mathrm{Mbits} / \mathrm{s}$ total network throughput in comparison to $100 \mathrm{Mbits} / \mathrm{s}$ for each link in the network. Thus, for these parameters the increased capacity from multiple links dominates over the diminished capacity due to guard times and blocking probability.

The effect of the link bandwidth on the delay-utilization characteristic can be also observed from (15). With a given network utilization, the delay is proportional to the link bandwidth. Intuitively, to keep the same utilization at a given $t_{\text {sion }}$, the time interval $t_{p}$ in cach time slot must also be same to have a same $\eta_{\text {siot }}$; therefore, the total bits transmitted in each slot is proportional to the link bandwidth. By (9), the compression delay $D$ will linearly increase as the link bandwidth increases. To overcome this effect, from (15), we see increasing the link bandwidth is the same as decreasing the idle time by the same proportion. As a result, smaller idle times (i.e., smaller network) can be used to counterbalance the increase in delay resulting from increases in the link bandwidth.

\section{HETEROGENEOUS TSS}

In Section IV, the homogeneous case where all circuits had the same bandwidth was considered. However, in practice we seek to integrate data, voice, and video traffic within a TSS network.

To illustrate the possibilities, consider the following assignment.

1) Each time slot is equivalent to a $16 \mathrm{Kbit} / \mathrm{s}$ channel.

2) Voice traffic uses a fixed $64 \mathrm{Kbit} / \mathrm{s}$ bit rate, and therefore each channel is assigned four (probably consecutive) time slots.

3) Interactive data traffic has a fixed rate, e.g., $16 \mathrm{Kbits} / \mathrm{s}$, $32 \mathrm{Kbits} / \mathrm{s}$ etc., and a number of slots is assigned depending on its bit ratc.

4) Video has no standard rate and is in the order of 20-200 Mbits/s. For simplicity, a whole frame circuit will be assigned for this large bandwidth traffic. Because the circuit switches are distributed and provide multiple circuits, this assignment will not significantly affect other traffic transmission.

5) Fixed length data such as file transfer can be assigned a certain number of slots based on its length. This will be explained in more detail in the remainder of this section.

Several characteristics of this approach should be mentioned as follows.

1) If it can be arranged for slots comprising a single channel to be contiguous, then there is a potential savings in guard time. In the extreme case of a high bandwidth full-motion video signal, one entire link or most of a link could be dedicated to one circuit. The overall utilization would then increase considerably above that estimated in the previous section.
2) For the heterogeneous case, the compression delay remains equal to one frame interval.

3) When the blocking probability or one circuit is small, say $10^{-3}$, the blocking probability for a higher rate circuit made up of lower rate circuits is approximately multiplied by the number of time slots assigned in a frame.

4) For file transfer, we simply want the maximum bandwidth available to minimize the time to transfer the file. If there is only one file transfer request, and the file can be transmitted within one frame by available time slots, the number of time slots assigned can be variable depending on the size of the file, and the blocking probability is not a meaningful concept. However, if there is more than one request for file transfer, or the file transfer cannot be finished in one frame, the maximum use of available bandwidth will possibly block others' requests. Thus, we should be conservative in assigning bandwidth for file transfer.

With regard to the last point on file transfer, we can suggest two approaches to assigning bandwidth. The first rule, the constant law.

$$
n=\left\lceil\frac{L_{D}}{t_{0} 16 \mathrm{Kbits} / \mathrm{s}}\right\rceil
$$

assigns a bandwidth proportional to the file length (resulting in a constant file transfer time). The second, the square root law, assigns a smaller bandwidth.

$$
n=\left\lceil\left(\frac{L_{D}}{L_{0}}\right)^{1 / 2} \frac{L_{0}}{t_{0} 16 \mathrm{Kbits} / \mathrm{s}}\right\rceil
$$

where $n$ is the number of time slots assigned for consecutive frames until the file is cxhausted, $L_{D}$ is the data size of the file, $t_{0}$ is a time parameter that is equal to the total transmission delay for the constant law, and $L_{0}$ is a length parameter. Transmission delay is equal to $m t_{0}$ when $L_{D}=m^{2} L_{0}$ at square root law, and $m$ is an integer. The resulting time for file transmission is

$$
\text { Transmission Delay } \approx\left\{\begin{array}{l}
t_{0}, \text { constant law: } \\
\left(\frac{L_{D}}{L_{0}}\right)^{1 / 2} t_{0}, \text { square root law. }
\end{array}\right.
$$

Numerical results are shown in Figs. 12 and 13. The constant law promises a smaller transmission delay (18) at the expense of larger blocking probability for other requests (by assigning more free time slots). The square root law has less effect on other users.

The performance and hardware costs are attractive for voice and video traffic in this heterogeneous TSS since they are well suited to circuit switching. The same can be said for file transfer activity as long as the file length is long enough that the circuit setup time is insignificant. For interactive data traffic, however, there are significant disadvantages as follows.

1) For very small data packets, the overhead in establishing a circuit is too large.

2) Interactive data traffic at a fixed rate is idle most of the time. The slots reserved are wasted during idle periods. This reduces the effective network utilization below our earlier estimates.

If these disadvantages are dominant, as in a network where interactive data represents a significant fraction of the total offered traffic, it is possible to overlay packet switching on top of TSS. For example, we can establish a packet network operating at lower speeds where the implementation costs are reasonable by establishing semipermanent circuit links through the TSS network. For example, a token ring can easily be established. TSS is very flexible in its ability to reconfigure 


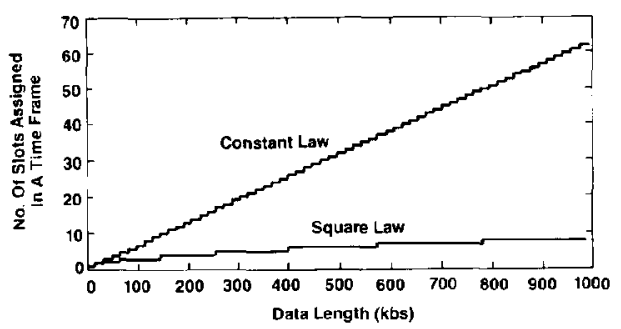

Fig. 12. Number of time slots assigned as a function of the file length for file transfer. Constant law provides constant transmission time (Fig. 13) by assigning time slots in a number linearly proportional to the file length. Square root law assigns fewer number of time slots for the same size of file, but with larger transmission delay. The tradeoffs is between the transmission delay and the blocking probability, which is approximately linearly proportional to the number of times slots assigned.

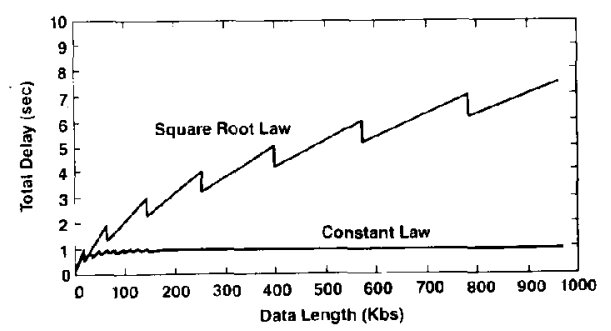

Fig. 13. Total time for file transmission as a function of the file length. Square root law has longer transmission time than constant law, but with a lower blocking probability.

and reassign capacity to various services such as packet data networks on a demand basis. It is also possible to combine TSS with a CSMA/CD data network in a portion of the frame [18], [19]. In fact, the presence of the active switches considerably simplifies the detection of collisions in such a network. We should emphasize again that due to the asynchronous nature of the switches, overlaid data networks can also use arbitrary bit rates as long as they adhere to the maximum rate imposed by the switch.

\section{Conclusion}

In this paper, we describe a new protocol for local-area networks: time slot switching or TSS. Since electronics is the factor limiting the bandwidth of practical optical fiber networks, TSS is very attractive because it minimizes electronics in switching nodes. It uses circuit switching, which appears more appropriate for very high speeds and is quite compatible with voice and video traffic, but does not preclude overlaid packet networks for interactive data traffic operating at more moderate speeds. The network is compatible with optical fibers technology, since it utilizes only point-to-point links at speeds that are quite modest by fiber standards. It is quite compatible with integrated traffic, and can easily provide different effective speeds for different services. Its greatest strength is the ability to reach quite impressive total throughputs without exotic technologies. Its greatest weakness is its limitation in geographical size to the order of one to two kilometers. This latter limitation can be overcome by the standard technique of adding gateways with buffering at the expense of additional delay.

With this switching scheme, TSS can be described as a " wide-band distributed PBX," and is perhaps a closer relative to today's PBX products than it is to traditional LAN approaches. Since technologies springing from both LAN and PBX products show promise in providing an integrated solution to local communications, it will be interesting to see which approach becomes dominant.

\section{APPENDIX A}

\section{Blocking Probability Analysis: Single Star}

In this appendix, the blocking probability for a single star shown in Fig. 4 is estimated based on Lee"s method [16]. In the following, we assume there are $N$ TCM's connected to the central switching node ( 4 in the figure) and $K$ time slots in each frame.

Assume that $p$, the probability of any one time slot on any one link being already used, is known, and that the events of different time slots on the same link or different links being used are independent. Now, suppose we want to form a new connection between two TCM's in any particular time slot. The probability of success is $(1-p)^{2}$. Therefore, the blocking probability for the connection in any particular time slot is $1-(1-p)^{2}$. Because any of $K$ time slots can be chosen for the connection, the probability that they are all blocked is

$$
P_{b k}=\left\{1-(1-p)^{2}\right\}^{K} .
$$

It remains to determine the probability $p$. Assume there are $A$ time slots in use on average and the traffic is uniformly distributed in all time slots. Then the active probability for each time slot is

$$
p=\frac{A}{N K} .
$$

since there are a total of $N K$ time slots among the $N$ TCM's.

\section{APPENDIX B}

\section{Blocking Analysis: DoUble StaR}

In this appendix, the blocking probability is estimated for the double star. In the following, we assume there are $\mathrm{N}_{2}$ substars connected to the central star and $N_{1}$ TCM's connected to each substar.

For the double star topology, a circuit connection can be within a substar or between two substars through the central star. If a circuit can be connected through the local substar, no connection will be required in the central star. Therefore, a blocking probability is larger if a circuit needs to go through the central star. To obtain a conservative estimate, this latter probability will be considered.

By Lee's method and using the same techniques as in Appendix A, the blocking probability is

$$
P_{b k}=\left\{1-\left(1-p_{1}\right)^{2}\left(1-p_{2}\right)^{2}\right\}^{K}
$$

where

$$
p_{1}=\frac{A}{K\left(N_{1}+1\right) N_{2}}
$$

is the probability of use for the time slots around local switches, and

$$
p_{2}=\frac{A}{K N_{2}}
$$

is that probability for the time slots around central switches.

Since $p_{1}$ is in general much smallest than $p_{2}$, the factor (1 $\left.p_{1}\right)^{2}$ in (B.1) can be neglected. Network throughput may therefore be expressed as

$$
A \approx K N_{2}\left\{1-\left(1-P_{b k}^{1 / k}\right)^{1 / 2}\right\}
$$




\section{REFERENCES}

[1] C. D. Tsao, "A LAN architecture overview," IEEE Commun., Mag., pp. 7-11, Aug. 1984.

[2] J. DeTreville and W. D. Sincoskie, "A distributed experimental communication system," IEEE Trans. Commun., to be published.

[3] J. DeTreville, "A simulation based comparison of voice transmission on CSMA/CD and token buses," AT\&T Bell Lab. Tech. J., pp. 3335, Jan. 1984

[4] T. A. Gonsalves, "Packet-voice communication on an Ethernet local computer network," XEROX CSL-82-5, Mar. 1982

[5] D. H. Johnson, G. C. O'Leary, "A local access network for packetized digital voice communication," IEEE Trans. Commun., pp. 679-688, May 1981 .

[6] J. O. Limb abd L. E. Flamm, "A distributed local area network packet protocol for combined voice and data transmission," IEEE J. Select. Areas Commun., pp. 926-934, Nov. 1983.

[7] B. Hailpern, A. Heller, L. W. Hoevel, and Y. J. Thefaine, "ALAN: A (circuit-switched) local area network," IEEE J. Select. Areas Commun., pp. 427-430, May 1985.

[8] U. Killat and J. Kruger, "Systems aspects and realization of wide-band switching in the local area," IEEE J. Select. Areas Commun., pp. 330-335, Mar. 1985

[9] H. J. Shin and D. A. Hodges, "A $250 \mathrm{Mb} / \mathrm{s}$ CMOS crosspoint switch," IEEE Int. Solid-State Circuits Conf., San Francisco, CA, Feb. 1988; also in Digest of Tech. Papers, pp. 114-115, 320-321.

[10] G. A. Hayward, A. M. Gottlieb, D. G. Boyer, and J. E. Berthold, "High speed $16 \times 16$ CMOS crosspoint switch," Electron. Lett., vol. 21, no. 20, pp. 923-925, 1985 .

[11] o. Enomoto et al., "Distributed microprocessors control architecture for versatile business communications," IEEE J. Select. Areas Commun., p. 508, July 1985 .

[12] A. Feiner, "Architecture, design, and development of the system 75 office communications system," IEEE J. Select. Areas Commun., p. 522, July 1985 .

[13] B. A. Burcz, "ASBU 501-A digital SPC voice/data switching system," IEEE J. Select. Areas Commun., p. 531, July 1985.

[14] J. M. Kasson and H. W. Johnson, "The CBX 11 switching architecture," IEEE J. Select, Areas Commun., p. 555, July 1985.

[15] K. Feher, Digital Communications, Satellite/Earth Station Engineering, S. J. Campanella and Daniel Schaefer, Eds. Englewood Cliffs, NJ: Prentice-Hall, 1981, Ch. 8.

[16] C. Y. Lee, "Analysis of switching networks," Bell Syst. Tech. J., pp. 1287-1315, Nov. 1955.

[17] Charles Clos, "A study of non-blocking switching networks," Bell Syst. Tech. J., pp. 406-427, Mar. 1953.

[18] M. -K. Liu and D. G. Messerschmitt, "A integrated network from TSS and CSMA/CD for data/voice/video LAN," to be published.

[19] M. -K. Liu, D. G. Messerschmitt, and D. A. Hodges, "An approach to fiber optics DATA/VOICE/VIDEO LAN, " in Proc. INFOCOM '86, pp. 516-523.

[20] W. A. Payne and H. S. Hinton, "System considerations for the lithium niobate photonic switching technology," Topic. Mect. Photon. Switch., Tech. Digest Series, vol. 13, 1987, pp. 74-76.

[21] J. M. Cooper, "The effect of cascading optical links without timing recovery for applications in local area networks," Master Rep., Univ. California, Berkeley, Fall 1986.

[22] H. J. Shin, Private Communications, Univ. California, Berkeley.

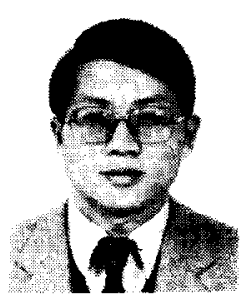

Ming-Kang Liu (S'80-M'87) was born in Taipei, Taiwan. He received the B.S.E.E. degree from National Taiwan University in 1981, and the M.S. and $\mathrm{Ph} . \mathrm{D}$. degrees in electrical engineering from University of California, Berkeley, in 1984 and 1987, respectively.

From 1981 to 1983 he was an Electrical Engineering Instructor at Chinese Naval Collcgc, Taiwan. Since 1987, he is a Member of Technical Staff of Bell Communications Research, Red Bank, NJ, where he is now working on a variety issues of broadband ISDN. His main research intercsts include high-specd fiber transmissions and switchings, and local area and metropolitan area networks.

David G. Messerschmitt (S'65-M'68-SM'78-F'83) He received the B.S degree from the University of Colorado in 1967, and the M.S. and Ph.D. degrees from the University of Michigan in 1968 and 1971, respectively.

$\mathrm{He}$ is a Professor of Electrical Engineering and Computer Sciences at the University of California at Berkeley. From 1968 to 1977 he was a Member of Technical Staff and later Supervisor at Bell Laboratories, Holmdel NJ, where he did systems engineering, development, and research on digital transmission and digital signal processing (particularly relating to speech processing). Current research interests include applications of digital signal processing, adaptive filtering, digital communications (on the subscriber loop and fiber optics), architecture and software approaches to programmable and dedicated hardware digital signal processing, communication network design and protocols, and computer-aided design of communications and signal processing systems. He has published over 100 papers and has 10 patents issued or pending in these fields. Since 1977 he has also served as a consultant to a number of companies.

Dr. Messerschmitt is a member of Eta Kappa Nu, Tau Beta Pi, Sigma Xi. and has several best paper awards. He has served as a Senior Editor of the IEEE COMMUNICATIONS MAGAZINE, as Editor for Transmission of the IEEE TRANSACTIONS ON COMMUNICATIONS, and as a member of the Board of Governors of the Communications Society. He has also organized and participated in a number of short courses and seminars devoted to continuing engineering education.

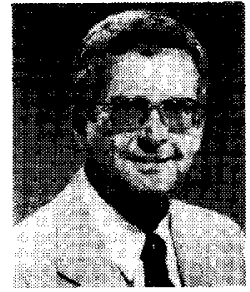

David A. Hodges (''59-M'65-SM'71-F'77) received the B.E.E. degree from Cornell University and the M.S. and Ph.D. degrees in electrical engineering from the University of California, Berkeley.

From 1966 to 1970 he worked at Bell Telephone Laboratories, first in the components area at Murray Hill, NJ, then as Head of the System Elements Research Department at Holmdel, NI. Currently, he is Professor of Electronical Engineering and Computer Sciences at the University of California, Berkeley, where he has been a member of the faculty since 1970 . He has been active in teaching and research on microelectronics technology and design and on communications and computer systems. Since 1984 he has led a growing research group at Berkeley on computer-integrated manufacturing systems.

Dr. Hodges is founding Editor of the new IEEE TRANSACTIONS ON SEMICONDUCTOR MANUFACTURING. He is a former Editor of the IEEE JOURNAL OF SOLID-STATE CIRCUITS and a past Chairman of the International Solid-State Circuits Conference. He is a member of the U.S. National Academy of Engineering. 